\title{
Associations between Prospective and Retrospective Subjective Memory Complaints and Neuropsychological Performance in Older Adults : The Finger Study
}

\section{Vaskivuo, Laura}

2018-11

Vaskivuo , L , Hokkanen , L , Hänninen , T , Antikainen , R , Bäckman , L, Laatikainen , T , Paajanen , T , Stigsdotter-Neely , A , Strandberg , T , Tuomilehto , J , Soininen , H , Kivipelto , M \& Ngandu , T 2018, ' Associations between Prospective and Retrospective Subjective Memory Complaints and Neuropsychological Performance in Older Adults : The Finger Study ', Journal of the International Neuropsychological Society, vol. 24 , no. 10 , pp. 1099-1109 . https://doi.org/10.1017/S135561771800053X

http://hdl.handle.net/10138/311856

https://doi.org/10.1017/S135561771800053X

unspecified

publishedVersion

Downloaded from Helda, University of Helsinki institutional repository.

This is an electronic reprint of the original article.

This reprint may differ from the original in pagination and typographic detail.

Please cite the original version. 


\title{
Associations between Prospective and Retrospective Subjective Memory Complaints and Neuropsychological Performance in Older Adults: The Finger Study
}

\author{
Laura Vaskivuo, ${ }^{1,2}$ Laura Hokkanen, ${ }^{2}$ Tuomo Hänninen, ${ }^{3}$ Riitta Antikainen, ${ }^{4,5}$ Lars Bäckman, ${ }^{6}$ Tiina Laatikainen, ${ }^{1,7,8}$ \\ Teemu Paajanen, ${ }^{9}$ Anna Stigsdotter-Neely, ${ }^{10}$ Timo Strandberg, ${ }^{4,11}$ Jaakko Tuomilehto, ${ }^{1,12,13}$ Hilkka Soininen, ${ }^{3,14}$ \\ Miia Kivipelto, ${ }^{1,14,15}$ AND Tiia Ngandu ${ }^{1,15}$ \\ ${ }^{1}$ Department of Public Health Solutions, Public Health Promotion Unit, National Institute for Health and Welfare, Helsinki, Finland \\ ${ }^{2}$ Department of Psychology and Logopedics, Faculty of Medicine, University of Helsinki, Finland \\ ${ }^{3}$ Neurocenter/ Neurology, Kuopio University Hospital, Finland \\ ${ }^{4}$ University of Oulu, Center for Life Course Health Research, Oulu, Finland \\ ${ }^{5}$ Medical Research Center Oulu, Oulu University Hospital and Oulu City Hospital, Oulu, Finland \\ ${ }^{6}$ Aging Research Center, Karolinska Institutet - Stockholm University, Stockholm, Sweden \\ ${ }^{7}$ Institute of Public Health and Clinical Nutrition, University of Eastern Finland; Kuopio, Finland \\ ${ }^{8}$ Hospital District of North Karelia, Joensuu, Finland \\ ${ }^{9}$ Finnish Institute of Occupational Health, Helsinki, Finland \\ ${ }^{10}$ Department of Psychology, Umeå University, Umeå, Sweden and Department of Social and Psychological Sciences, Karlstad University, Karlstad, Sweden \\ ${ }^{11}$ University of Helsinki, Clinicum, and Helsinki University Hospital, Helsinki, Finland \\ ${ }^{12}$ South Ostrobothnia Central Hospital, Seinäjoki, Finland and Department of Public Health, University of Helsinki, Helsinki, Finland \\ ${ }^{13}$ Dasman Diabetes Institute, Kuwait City, Kuwait, Danube University Krems, Krems, Austria and Kind Abdulaziz University, Jeddah, Saudi Arabia \\ ${ }^{14}$ Institute of Clinical Medicine/Neurology, University of Eastern Finland, Kuopio, Finland \\ ${ }^{15}$ Division of Clinical Geriatrics, Center for Alzheimer Research, NVS, Karolinska Institutet, Stockholm, Sweden \\ (Received January 20, 2018; Final Revision May 24, 2018; Accepted May 28, 2018; First Published Online September 4, 2018)
}

\begin{abstract}
Objectives: Subjective memory complaints (SMCs) are among the key concerns in the elderly, but their role in detecting objective cognitive problems is unclear. The aim of this study was to clarify the association between SMCs (both prospective and retrospective memory complaints) and neuropsychological test performance in older adults at risk of cognitive decline. Methods: This investigation is part of the FINGER project, a multicenter randomized controlled trial aiming at preventing cognitive decline in high-risk individuals. The cognitive assessment of participants was conducted at baseline using a modified neuropsychological test battery (NTB). SMCs were evaluated with the Prospective and Retrospective Memory Questionnaire (PRMQ) in a sub-sample of 560 participants (mean age, 69.9 years). Results: Having more prospective SMCs was associated with slower processing speed, but not with other NTB domains. Retrospective SMCs were linked to poorer function on NTB total score, processing speed, and memory. Executive function domain was not associated with any PRMQ ratings. Depressive symptoms and poor quality of life diluted the observed associations for NTB total score and memory. However, the association between PRMQ and processing speed remained even after full adjustments. Conclusions: Our results indicate that self-reported memory problems, measured with PRMQ, are associated with objectively measured cognitive performance. Such complaints in healthy elderly people also seem to reflect reduced mental tempo, rather than memory deficits. Slowing of processing speed may thus be negatively related to memory selfefficacy. It is also important to consider affective factors among those who report memory problems. (JINS, 2018, 24,
\end{abstract} 1099-1109)

Keywords: Subjective memory complaints, Prospective memory, Retrospective memory, Cognitive performance, Memory, Processing speed

\section{INTRODUCTION}

Subjective memory complaints (SMCs) are usual concerns

*Correspondence and reprint requests to: Laura Vaskivuo, Department of Public Health Solutions, Public Health Promotion Unit, National Institute for Health and Welfare, P.O 30, 00271 Helsinki, Finland. E-mail: laura.vaskivuo @helsinki.fi expressed by old people, but not all complainers have deficits on objective cognitive testing. SMCs are highly prevalent among older persons and their prevalence increases with age (Iliffe \& Pealing, 2010). According to one study $43 \%$ of 
people aged 65-74 years reported SMCs, whereas the prevalence was as high as $88 \%$ in those older than 85 years (Larrabee \& Crook, 1994). With the aging of populations the number of persons with dementia will increase rapidly (Alzheimer's Disease International, 2015). The relationship between SMCs and objective cognitive performance is pivotal in the early detection of dementia and Alzheimer's disease (AD), and in identifying people for early preventive interventions and disease-modifying therapies.

Findings on associations between SMCs and actual concurrent or future cognitive functioning are mixed. In some studies, SMCs have been related to worse objective neuropsychological performance (Amariglio et al., 2012; Hohman, Beason-held, Lamar, \& Resnick, 2011; Jacinto, Brucki, Porto, Martins, \& Nitrini, 2014; Reid \& MacLullich, 2006), brain changes occurring in early AD (Jessen et al., 2006; Meiberth et al., 2015; Schneider, Arvanitakis, Leurgans, \& Bennett, 2009) and prodromal stages of AD (Lehrner et al., 2014), but in other studies such relationships have not been found (Iliffe \& Pealing, 2010; Jungwirth et al., 2004; Studer, Donati, Popp, \& von Gunten, 2014). According to a recent meta-analysis (Mitchell, Beaumont, Ferguson, Yadegarfar, \& Stubbs, 2014), older people with SMCs but without objective cognitive problems are twice as likely to develop dementia compared with those without SMCs.

Differences between studies may to some extent be explained by differences in study population characteristics and methodological factors (Crumley, Stetler, \& Horhota, 2014). Longer questionnaires assessing memory abilities yield larger relationships with objective memory performance (Crumley et al., 2014). Little is known as to how SMCs relate to cognitive domains other than memory. Steinberg and colleagues (2013) suggested that lower frequency of SMCs may be associated with better scores on both memory and executive function tasks.

The interrelationships among SMCs, depression, and cognition are complex (Iliffe \& Pealing, 2010; Reid \& MacLullich, 2006; Rönnlund, Vestergren, Mäntylä, \& Nilsson, 2011) and SMCs may relate to affective and personality traits more than to actual memory performance (Hänninen et al., 1994; Reid \& MacLullich, 2006; Steinberg et al., 2013). SMCs have also been found to be associated with poorer quality of life (Mol et al., 2007; Montejo, Montenegro, Fernandez, \& Maestu, 2011; Steinberg et al., 2013), older age, female sex, lower education (Iliffe \& Pealing, 2010), poorer perceived health, and less physical activity (Lee, 2014; Sargent-Cox, Cherbuin, Sachdev, \& Anstey, 2011). Furthermore, SMCs have been proposed to reflect low memory self-efficacy beliefs rather than memory impairment (Ponds \& Jolles, 1996). In some studies, APOE $\varepsilon 4$ allele has been associated with increased SMCs and subjective cognitive concerns (Samieri et al., 2014; Small et al., 1999; Stewart et al., 2001).

Studies of aging-related cognitive changes and dementia have typically focused on retrospective memory (RM), which involves remembering learned information or events that have happened in the past, and its decline both with age and in $\mathrm{AD}$ is well documented (Maylor, Smith, Della Sala, \& Logie, 2002). Prospective memory (PM), the ability to remember to perform a planned action or intention in the future (McDaniel \& Einstein, 2011) has been shown to be an important determinant of independent living (Bisiacchi, Tarantino, \& Ciccola, 2008; Crumley et al., 2014; Kliegel \& Martin, 2003; Salthouse, Berish, \& Siedlecki, 2004; Woods, Weinborn, Velnoweth, Rooney \& Bucks, 2012), and contribute to prediction of AD (Jones, Livner, \& Bäckman, 2006).

Some previous studies have reported that people have more subjective PM than RM complaints (Crawford, Smith, Maylor, Della Sala, \& Logie, 2003; Kliegel \& Jager, 2006; Piauilino et al., 2010; Rönnlund, Mäntyla, \& Nilsson, 2008). However, studies on the relationship between SMCs and cognitive function have usually not assessed PM and RM complaints separately. Studies where this has been done have found stronger links for PM compared with RM (Crumley et al., 2014; Zeintl, Kliegel, Rast, \& Zimprich, 2006). In one study, however, (Rönnlund et al., 2011) in a population-based sample of older adults subjective PM and RM problems did not show relationships with objective memory abilities or general cognitive functioning as assessed by the Mini-Mental State Examination (MMSE). In another study, Eschen, Martin, Gasser, and Kliegel (2009) found associations between both subjective PM and RM complaints and executive function in healthy older adults.

The relationship between SMCs and objective cognitive performance remains unclear. The aim of this study is to examine the association between different types of SMCs (PM and $\mathrm{RM}$ ) and neuropsychological test performance in different cognitive functions (global cognition, executive functions, processing speed, and memory) in older adults at risk of cognitive decline. A large number of variables (e.g., demographic factors, APOE $\varepsilon 4$ status and self-reported measures of depressive symptoms, quality of life, subjective health, and physical activity) are taken into account as potential confounding factors.

\section{METHODS}

\section{Study Design}

The current study is part of the Finnish Geriatric Intervention Study to Prevent Cognitive Impairment and Disability (FINGER). The protocol and main results from the FINGER project have been described elsewhere (Kivipelto et al., 2013; Ngandu et al., 2015). In brief, FINGER is a multi-center randomized controlled trial aiming to lower the risk of cognitive impairment in older adults at increased risk of cognitive decline (ClinTrials identifier NCT01041989). The intervention consisted of nutritional guidance, exercise, cognitive training, social activity, and management of metabolic and vascular risk factors. The control group received regular health advice. The primary outcome was cognitive 
performance. FINGER has been approved by the Coordinating Ethics Committee of the Helsinki and Uusimaa Hospital District. Human data included were obtained in compliance with the Helsinki Declaration.

\section{Participants}

Participants were 60-77 years old at the start of the study. They were recruited from previous random, populationbased, non-intervention surveys that can be considered representing well the Finnish population (Saaristo et al., 2007; Vartiainen et al., 2010). Participants were prescreened with the CAIDE Dementia Risk Score (Kivipelto, 2006), and those scoring at least 6 points were further screened with the Consortium to Establish a Registry for Alzheimer's Disease (CERAD) neuropsychological test battery (Morris, 1988).

\section{Inclusion and Exclusion Criteria}

For inclusion, people had to fulfill also at least one of the following criteria: (1) Word List Memory Task learning score (10 words $\times 3$ ) $\leq 19$ words; (2) Word List Recall $\leq 75 \%$; or (3) MMSE (Folstein, Folstein, \& McHugh, 1975) $\leq 26 / 30$ points. These criteria selected persons with cognitive performance at the mean level or slightly lower than expected for age according to Finnish population norms (Hänninen et al., 2010), but without substantial cognitive impairment (Ngandu et al., 2014).

Exclusion criteria were conditions affecting safe engagement in the intervention: malignant diseases; major depression; previously diagnosed dementia/substantial cognitive deficits; suspected dementia after clinical assessment by study physician at screening visit; MMSE less than 20 points; symptomatic cardiovascular disease; revascularization within 1 year; severe loss of vision, hearing, or communicative ability; conditions preventing cooperation as judged by the study physician; as well as coincident participation in any other intervention trial (Ngandu et al., 2014).

Screening was completed in December 2011 when the intended recruitment target $(n=1260)$ was achieved. After completing the baseline measurements, randomization into intervention or control groups was performed. These groups were equal in size (Kivipelto et al., 2013).

To measure SMCs, The Prospective and Retrospective Memory Questionnaire (PRMQ) was added to the study protocol after study onset, and this information is thus available for participants $(n=560)$ who were included after August 2010.

\section{Cognitive Assessment}

Cognitive performance was measured at baseline using a modified Neuropsychological Test Battery (NTB) known to be a reliable and sensitive measure for mild cognitive changes (Harrison et al., 2007). Additional tasks to detect also executive dysfunction were included. The assessment was made by trained psychologists. All baseline information was collected before randomization.

The NTB consisted of 14 tests that represent three different cognitive domains: The memory domain included Visual
Paired Associates immediate (score range, 0-18) and delayed (score range, 0-6); Logical Memory immediate (score range, 0-25) and delayed (score range, 0-25) of the Wechsler Memory Scale-Revised (WMS-R) (Wechsler, 1998); and Word List Learning (score range, 0-30) and Delayed Recall (score range, 0-10) from the CERAD test battery (Morris, 1988). The executive function domain included Category Fluency (Morris, 1988), Digit Span (Wechsler, 1998), Concept Shifting (CST; Van der Elst, Van Boxtel, Van Breukelen, \& Jolles, 2006a) (condition C), Trail Making Test (TMT), shifting score B-A (Reitan, 1958), and a shortened 40-item version of the original Stroop test, interference score from condition 3-2 (Golden, 1978). The processing speed domain included Letter Digit Substitution (van Der Elst, van Boxtel, van Breukelen, \& Jolles, 2006b), Concept Shifting (condition A), and Stroop (condition 2).

Zero-skewness log-transformation was applied to skewed NTB components, and standardized Z-scores were calculated for each test using the mean and standard deviation of the current population. NTB total score and domain scores for executive functioning, processing speed and memory were obtained by averaging individual NTB component $Z$-scores. Because of missing values the minimum number of required NTB components was set to $8 / 14$ for calculating NTB total score, $3 / 5$ for executive functioning, $2 / 3$ for processing speed, and $3 / 6$ for memory.

In addition to the NTB, a PM task (PMT), modified from the Rivermead Behavioural Memory Test (Wilson, Cockburn, \& Baddeley, 1985) was used. In PMT, at the beginning of the test session, participants were told to remind the psychologist that he/she must give a binder from the table to them at the end of the session. The scoring was as follows: remembering the task without a cue $($ score $=3)$, remembering with one cue $($ score $=2)$, remembering with two cues $($ score $=1)$ or not remembering at all $($ score $=0)$. In the analyses, PMT was divided into two groups: $\leq 2$ or 3 points.

\section{Assessment of Subjective Memory}

PRMQ is a 16-item self-report measure of memory failures in everyday life, the only instrument available that assesses subjective PM and RM in equal depth (Crawford et al., 2003). Eight of the items enquire about PM and eight about RM. PRMQ has been shown to have acceptable reliability and validity (Crawford et al., 2003; Rönnlund et al., 2008). Participants evaluate how often each of the situations occur in everyday life on a 5-point scale: very often, quite often, sometimes, rarely, and never. The minimum total score is 16 , and the maximum is 80 . Higher scores indicate more SMCs. PRMQ was translated into Finnish by the research team. Participants completed the PRMQ at 6-month visits. Those $(n=26)$ who did not complete the whole PRMQ were excluded from analyses.

\section{Other Questionnaires and Measures}

Subjects completed several questionnaires inquiring about lifestyles, health status, mood, and quality of life at the 
baseline visits. Depressive symptoms were assessed by the Zung Self-Rating Depression Scale (Zung, 1965), which includes 20 items scored from 1 to 4 . The range is 20 to 80 , with higher scores indicating more depressive symptoms. Quality of life was evaluated by the RAND-36, which includes 36 items assessing eight aspects of health-related quality of life. A summary score is derived from those eight scales. The questionnaire is validated in the general Finnish population (Aalto, Aro, \& Teperi, 1999).

Data on subjective health status were collected using a 5 -point Likert-scale ( $1=$ very good; $5=$ very poor). Selfreported frequency of leisure-time physical activities causing sweating and breathlessness lasting at least $20 \mathrm{~min}$ at least twice a week was regarded as being active. Education was measured as number of years of schooling. Genomic DNA was extracted from venous blood samples with Chemagic MSM1 from PerkinElmer using magnetic beads. APOE genotyping was determined by polymerase chain reaction using TaqMan genotyping assays [Applied Biosystems (ABI), Foster City, CA] for two single-nucleotide polymorphisms (rs429358 and rs7412) and an allelic discrimination method on the ABI 7500 platform (De La Vega, Lazaruk, Rhodes, \& Wenz, 2005).

\section{Statistical Analyses}

All statistical analyses were performed using IBM SPSS Statistics version 22. Level of significance was set at $p=.05$ in all analyses.

Descriptive statistics were used to characterize the study population. First, the NTB scores at baseline were compared in relation to PRMQ scores at six months. Bivariate associations between participant characteristics and PRMQ scores were analyzed using Pearson correlation coefficients, $t$ tests, and analysis of variance as appropriate. Linear (NTB and its subdomains) or logistic (PMT) regression models were used to analyze associations between neuropsychological performance and PRMQ scores. NTB domains and PMT were entered as dependent variables and PRMQ (total, PM, and RM) as independent variables. Variables considered as potential confounders based on prior work and/or observed bivariate associations were introduced into the analyses as follows: model 1 included age, sex, study site, and years of education.

In model 2, the Zung depression scale was added; in model 3, marital status (married/cohabiting), subjective health, and physical activity were also entered; and in the final model 4 , $A P O E$ status ( $\varepsilon 4 v s$. non $\varepsilon 4$ carrier) was added. Because of a strong correlation between the Zung depression scale and quality of life (RAND36), the latter was modeled in a separate regression analysis with two blocks. The first model was adjusted by a block including age, sex, study site, education, and quality of life, and the second model included a block with full adjustments (without depression score).

\section{RESULTS}

\section{Characteristics of the Study Population}

There were 560 participants ( 284 men; 276 women). The age range was 60.8-79.8 (men: mean age $=69.2$ years, $S D=4.92$; women: mean age $=70.5$ years, $S D=4.8$ ). The mean score on the total PRMQ was $36.30(n=560 ; S D=7.9$; range, $16-66)$. For the prospective subdomain, the mean was $18.0(S D=4.3$;

Table 1. Baseline characteristics of the participants

\begin{tabular}{|c|c|c|c|}
\hline Characteristics at baseline & $N$ & $\mathrm{M} / \%$ & $S D$ \\
\hline \multicolumn{4}{|l|}{ Demographic characteristics } \\
\hline Age y & 560 & 69.9 & 4.9 \\
\hline Sex (\% men/ women $)$ & 560 & $50.7 / 49.3$ & \\
\hline Education $(\mathrm{y})$ & 559 & 10.0 & 3.4 \\
\hline Married / cohabiting (\%) & 559 & 72.5 & \\
\hline \multicolumn{4}{|l|}{ Self-reported measures } \\
\hline Zung self-rating depression scale & 502 & 34.1 & 7.6 \\
\hline Quality of life (RAND-36) & 495 & 75.8 & 17.9 \\
\hline Subjective health (quite good or very good, \%) & 557 & 58.2 & \\
\hline Physically active (\%) & 553 & 72.3 & \\
\hline \multicolumn{4}{|l|}{ PRMQ } \\
\hline Total score & 560 & 36.3 & 7.9 \\
\hline Prospective memory subscale & 560 & 18.0 & 4.3 \\
\hline Retrospective memory subscale & 560 & 18.3 & 4.2 \\
\hline \multicolumn{4}{|l|}{ Other } \\
\hline \multicolumn{4}{|l|}{ Study site: } \\
\hline Helsinki & 72 & 12.9 & \\
\hline Vantaa & 84 & 15.0 & \\
\hline Kuopio & 201 & 35.9 & \\
\hline Turku & 203 & 36.3 & \\
\hline APOE $\varepsilon 4$ carriers & 169 & 32.4 & \\
\hline
\end{tabular}


Table 2. Cognitive performance at baseline

\begin{tabular}{|c|c|c|c|c|}
\hline Neuropsychological test & & $n$ & Mean & $S D$ \\
\hline \multicolumn{5}{|l|}{ Memory } \\
\hline WMS-R Logical Memory (immediate) & & 558 & 10.9 & 3.5 \\
\hline WMS-R Logical Memory (delayed) & & 558 & 9.3 & 3.7 \\
\hline CERAD Word List Learning & & 558 & 18.1 & 3.2 \\
\hline CERAD Word List Recall & & 557 & 5.4 & 1.7 \\
\hline WMS-R Visual Paired Associates (immediate) & & 553 & 9.4 & 3.6 \\
\hline WMS-R Visual Paired Associates (delayed) & & 552 & 3.4 & 1.8 \\
\hline \multicolumn{5}{|l|}{ Executive Function } \\
\hline CERAD Category Fluency & & 558 & 22.2 & 5.6 \\
\hline WMS-R Digit Span (total) & & 558 & 11.4 & 2.9 \\
\hline CST $\left(\right.$ condition C) ${ }^{\mathrm{a}}$ & & 532 & 64.8 & 36.7 \\
\hline TMT shifting score $(\mathrm{B}-\mathrm{A})^{\mathrm{a}}$ & & 533 & 105.7 & 65.4 \\
\hline Stroop test interference score $(3-2)^{\mathrm{a}}$ & & 556 & 35.3 & 20.2 \\
\hline \multicolumn{5}{|l|}{ Processing speed } \\
\hline LDST & & 558 & 21.6 & 5.7 \\
\hline $\operatorname{CST}(\text { condition } \mathrm{A})^{\mathrm{a}}$ & & 558 & 32.8 & 9.4 \\
\hline Stroop test (condition 2$)^{\mathrm{a}}$ & & 557 & 29.2 & 6.5 \\
\hline \multicolumn{5}{|l|}{ Other } \\
\hline \multirow[t]{2}{*}{ Prospective memory task (\%) } & $2 \leq$ points & 200 & 36.3 & \\
\hline & 3 points & 351 & 63.7 & \\
\hline MMSE & & 559 & 27.0 & 2.2 \\
\hline
\end{tabular}

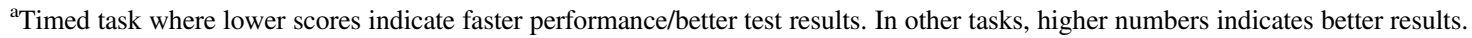

WMS-R = Wechsler Memory Scale-Revised, CERAD = Consortium to Establish a Registry for Alzheimer's Disease, CST $=$ Concept Shifting Test, TMT $=$ Trail Making Test, LDST = Letter Digit Substitution Test, MMSE = Mini-Mental State Examination

range, 8-34), and for the retrospective subdomain, the mean was $18.3(S D=4.2$; range 8-33). Thus, complaints were quite comparable for PM and RM. Subject characteristics are reported in Table 1 and their cognitive performance in Table 2.

In bivariate analyses, higher total PRMQ ratings were associated with more depressive symptoms, lower quality of life, and worse subjective health. Higher PM ratings were associated with more depressive symptoms, lower quality of life, physical inactivity, and worse subjective health. Higher RM ratings were linked to more depressive symptoms, poorer quality of life, lower education, older age, and worse subjective health.

\section{SMCs and Neuropsychological Performance}

Results from the linear regression models (regression coefficients $\beta$ and $p$-values) and logistic regression models (estimated odds ratios) are presented in Tables 3 and 4. Having more subjective complaints was related to poorer performance on the cognitive tests. Total PRMQ scores were associated with NTB total and slower processing speed, but not with other NTB domains or the PMT (Table 3). The associations were significant after controlling for the effects of age, sex, study site, and education. After controlling for depression, the association with total NTB was no longer significant. By contrast, the association with processing speed remained significant after full adjustments (model 4 ).

In the models adjusted with demographics and quality of life, PRMQ total score was only associated with processing speed. This association also remained after full adjustments (Table 4).

\section{Subjective PM Complaints and Neuropsychological Performance}

PM problems were associated with slower processing speed, but not with NTB total, memory, executive function, or the PMT. The association with processing speed remained after full adjustments (Table 3 ) and also in the model adjusted for quality of life (Table 4).

\section{Subjective RM Complaints and Neuropsychological Performance}

Perceived RM problems were associated with poorer function on the NTB total, processing speed, and memory after controlling for the effects of sex, age, study site, and education (Table 3 and Table 4). Depression and quality of life diluted the observed associations with NTB total and memory. The association with slower processing speed remained nearly significant $(p=.051)$ after adjusting for age, sex, study site, education, depression, marital status, subjective health, and physical activity. Executive function domain as well as the PMT were not related to perceived RM problems.

\section{DISCUSSION}

Attempts to identify early signs of memory disorders have increased the interest in studying SMCs. Yet, the association between SMCs and objective cognitive performance is still controversial. The aim of this study was to clarify the 
Table 3. Relationships (regression coefficients $[\beta]$ and $p$ values) of PRMQ (total score and subdomains) to NTB and prospective memory performance (PMT), adjusted for confounding factors

\begin{tabular}{lcccc}
\hline \hline & Model 1 & Model 2 & Model 3 & Model 4 \\
\hline Total PRMQ complaints & & & & \\
$\quad$ NTB total & $-0.003(0.021)$ & $-0.002(0.224)$ & $-0.002(0.146)$ & $-0.003(0.076)$ \\
Processing speed & $-0.008(0.000)$ & $-0.005(0.019)$ & $-0.006(0.015)$ & $-0.006(0.018)$ \\
Executive functions & $-0.002(0.366)$ & $-0.001(0.806)$ & $-0.002(0.473)$ & $-0.002(0.454)$ \\
Memory & $-0.002(0.248)$ & $-0.001(0.484)$ & $-0.001(0.534)$ & $-0.002(0.260)$ \\
PMT $^{\mathrm{a}}$ & $0.992(0.482)$ & $1.005(0.684)$ & $1.002(0.857)$ & $1.004(0.745)$ \\
Prospective memory complaints & & & \\
$\quad$ NTB total & $-0.004(0.059)$ & $-0.002(0.526)$ & $-0.002(0.397)$ & $-0.003(0.227)$ \\
Processing speed & $-0.015(0.000)$ & $-0.009(0.033)$ & $-0.009(0.025)$ & $-0.009(0.026)$ \\
Executive functions & $-0.004(0.282)$ & $-0.001(0.870)$ & $-0.002(0.583)$ & $-0.003(0.513)$ \\
Memory & $-0.001(0.838)$ & $0.001(0.726)$ & $0.001(0.680)$ & $0.000(0.933)$ \\
PMT & $0.972(0.172)$ & $0.995(0.827)$ & $0.991(0.708)$ & $0.997(0.890)$ \\
Retrospective memory complaints & & & \\
$\quad$ NTB total & $-0.006(0.012)$ & $-0.004(0.142)$ & $-0.004(0.101)$ & $-0.005(0.063)$ \\
Processing speed & $-0.014(0.000)$ & $-0.009(0.040)$ & $-0.009(0.037)$ & $-0.008(0.051)$ \\
Executive functions & $-0.003(0.458)$ & $0.000(0.954)$ & $-0.002(0.651)$ & $-0.002(0.673)$ \\
Memory & $-0.005(0.047)$ & $-0.005(0.109)$ & $-0.005(0.134)$ & $-0.006(0.056)$ \\
PMT & $1.002(0.938)$ & $1.024(0.329)$ & $1.018(0.474)$ & $1.019(0.459)$ \\
\hline \hline
\end{tabular}

Note. PMT results are based on logistic regression models, all other data are based on linear regressions. Model 1 adjusted with age, sex, study site. and education. Model 2 adjusted with age, sex, study site, education. and depression. Model 3 adjusted with age, sex, study site, education, depression, marital status, subjective health. and physical activity. Model 4 adjusted with age, sex, study site, education, depression, marital status, subjective health, physical activity, and APOE4 allele.

${ }^{\mathrm{a} C}$ Coefficients are estimated odds ratio from logistic regression models.

Table 4. Relationships (regression coefficients $[\beta]$ and $p$ values) of PRMQ (total score and subdomains) to NTB and the PMT adjusted for confounding factors including quality of life

\begin{tabular}{lcc}
\hline \hline & Model 1 & Model 2 \\
\hline Total PRMQ complaints & & \\
$\quad$ Total NTB & $-0.002(0.209)$ & $-0.002(0.075)$ \\
Processing speed & $-0.005(0.030)$ & $-0.005(0.028)$ \\
Executive functions & $-0.001(0.783)$ & $-0.002(0.431)$ \\
Memory $_{\text {PMT }^{\mathrm{a}}}$ & $-0.001(0.451)$ & $-0.002(0.256)$ \\
Prospective memory complaints & $0.993(0.605)$ & $0.993(0.580)$ \\
Total NTB & $-0.002(0.398)$ & $-0.004(0.156)$ \\
Processing speed & $-0.008(0.048)$ & $-0.008(0.046)$ \\
Executive functions & $-0.002(0.595)$ & $-0.004(0.297)$ \\
Memory & $0.000(0.869)$ & $-0.001(0.781)$ \\
PMT & $0.974(0.262)$ & $0.977(0.329)$ \\
Retrospective memory complaints & & \\
Total NTB & $-0.004(0.159)$ & $-0.005(0.083)$ \\
Processing speed & $-0.007(0.080)$ & $-0.007(0.090)$ \\
Executive functions & $0.001(0.865)$ & $-0.001(0.830)$ \\
Memory & $-0.005(0.098)$ & $-0.006(0.062)$ \\
PMT & $1.004(0.855)$ & $0.999(0.972)$ \\
\hline
\end{tabular}

Note. The PMT results are based on logistic regression models, the others are based on linear regressions. Model 1 adjusted with age, sex, study site, education, and quality of life.

Model 2 adjusted with age, sex, study site, education, quality of life, marital status, subjective health, physical activity, and APOE4 allele.

${ }^{\mathrm{a} C}$ Coefficients are estimated OR from logistic regression model. relationship between SMCs and cognitive performance in older adults at risk of cognitive decline, with special focus on the role of PM and RM memory complaints.

SMCs, as assessed by the PRMQ, were associated with objectively measured cognitive performance. More specifically, the PRMQ total score was linked to total NTB and processing speed. PM subscale showed associations with processing speed only while RM subscale showed associations with all measured cognitive domains except for executive function. In line with much previous research depressive symptoms and quality of life diluted several of these associations. Importantly, however, the association between PRMQ and processing speed remained after full adjustments.

The fact that SMCs showed the strongest association with processing speed was somewhat surprising, as the questionnaire items did not specifically focus on mental tempo. Although the magnitude of age-related cognitive deficits vary considerably across individuals, reduced processing speed is strongly related to age (Salthouse, 2000), and accounts for significant parts of impairment across multiple cognitive domains (Finkel, McArdle, Reynolds, \& Pedersen, 2007; Lemke \& Zimprich, 2005). Reduced processing speed has also been shown to be associated with changes in brain white matter integrity (Kuznetsova, et al., 2016; Penke et al., 2010), as well as hippocampal integrity (Aribisala et al., 2014) and may thus represent early steps of neurodegenerative processes. Processing speed also plays an important role for functioning in everyday life and health status in aging 
(Owsley, Sloane, McGwin, \& Ball, 2002; Rosnick, Small, Graves, \& Mortimer, 2004).

The current data suggest that SMCs are more a sign of slowing down rather than reflecting memory problems in this cognitively fairly healthy population. It is possible that SMCs might be the first signals of cognitive impairment even in this population; however, longer follow-up studies are still needed to verify how large portion of subjects suffering from SMCs will later progress to neurodegenerative diseases. People express different kinds of cognitive difficulties often in terms of memory failures. Slowing may thus be negatively linked to cognitive self-efficacy. Current societal demands where time-limited performance is increasingly important for functional independence and quality of life further underscores the importance of processing speed.

After adjustments for several potential confounding factors, we found a significant association between the RM subscale and memory. Adding APOE to the last model diluted the effect a bit suggesting a biological component to both RM and memory performance. Neither total PRMQ scores nor the PM subscale showed a similar relationships to memory. In a meta-analysis Crumley et al. (2014), the relationship between SMCs and objective memory performance was varied and inconclusive. In that analysis, several demographic and study characteristics were related to the strength of the association. The absence of widespread associations between SMCs and objective memory performance may partly be due to the methods used. Commonly used memory tests as those used in our study are retrospective in nature and may tap RM complaints more accurately than PM complaints. If SMCs would include PM complaints, associations may be better examined using objective PM measures rather than traditional RM measures. That said, we included a single PMT, which alone failed to show associations with PRMQ.

In past research, executive functions have shown associations with PM complaints (Eschen et al., 2009; Steinberg et al., 2013). This may reflect the fact that PM problems are more easily observed in everyday life, and that PMTs rely more on executive processes than RM tasks (Mäntylä, 2003). Our study did not confirm this notion. We did not find associations between executive functioning and the PM subscale or to total PRMQ score. The mixed evidence may partly reflect methodological differences. Many different tests are used to measure executive functions and the effect of processing speed is not always separated from the outcome.

Eschen et al. (2009) found PM scores to be related to one out of three executive function tests (3rd plate Victoria-Stoop Test), but not with two other tests (Digit Span Backward and TMT-B/A). In another study, PRMQ scores were related to executive function, evaluated with subtest of CogState computerized cognitive test battery (Groton Maze learning task; Steinberg et al., 2013). We analyzed executive function as a cognitive domain score including five different tests and considered processing speed separately. It may also be that there are executive processes that are missed by our standard measures. One study (McDaniel \& Einstein, 2011) highlighted several components of PM, and hypothesized that some are more related to frontal functioning whereas others rely more on medial-temporal regions. Thus, measurements capturing the full range of executive processes potentially related to PM performance are warranted (Martin, Kliegel, \& Mcdaniel, 2003).

The role of depression is important in SMCs. Among elderly people, depressive symptoms are common and their interrelationship with cognition is complex. In earlier studies with healthy elderly persons, findings have been mixed. SMCs have been found to relate more to depression or poor quality of life than to objective cognitive performance. One study (Rönnlund et al., 2011) examined predictors of SMCs using PRMQ and found SMCs to reflect more strongly mood state and personality factors than objective memory performance.

In the present study, affective factors diluted the observed associations with all cognitive domains, except processing speed. That particular association remained even after full adjustments. Whether SMCs are symptoms of depression or whether depression is a consequence of worries about subjectively perceived memory problems remains to be determined (Crumley et al., 2014; Iliffe \& Pealing, 2010). Often, associations between SMCs and objective memory impairment have disappeared after adjusting for depression (e.g., Iliffe \& Pealing, 2010; Jorm et al., 2004). However, in some studies SMCs have been related to cognitive status, independent of depressive symptoms (Lehrner et al., 2014; Reid \& MacLullich, 2006). Conceivably, variability of assessment methods for SMCs, depression, and cognition may be a key reason behind these inconsistent findings in this domain of inquiry (Reid \& MacLullich, 2006).

Most previous studies using PRMQ in healthy populations indicate that perceived PM failures are more common than RM memory failures (Crawford et al., 2003; Kliegel \& Jager, 2006; Mäntylä, 2003). Contrary to those studies, but in line with the results of Eschen et al. (2009), subjective PM and RM deficits were equally expressed in our study population. Age might be one possible factor behind the contradictory findings, as our sample as well as that in the study by Eschen et al. (2009) were somewhat older than samples in the other studies. Transition from working life to retirement and the use of external memory aids may reduce PM requirements in everyday life among older adults.

In addition to age, also other variables, such as affective and personality variables matter (Kliegel \& Zimprich, 2005) and PM performance has been suggested to constitute a reliable predictor of PM complaints only when depressive symptoms are low (Zeintl et al., 2006). It has also been suggested that the length of the method used may play a role. Longer assessments may reflect SMCs more accurately than shorter assessments (Crumley et al., 2014). PRMQ is a 16-item self-report measure assessing PM and RM failures in everyday life.

This study has several strengths. The study population is drawn from earlier population-based studies, and with the inclusion criteria used, it is representative of the normal Finnish population with some risk factors for dementia and 
cognitive function at the average or slightly below the average level of this age group (Hänninen et al., 2010). Self-reported cognitive concerns become less reliable as individuals progress toward dementia (Buckley et al., 2015; Jorm, Christensen, Korten, Jacomb, \& Henderson, 2001). Our inclusion criteria for cognition at the screening visit were used to select a high-risk population, and to exclude both the individuals whose cognitive performance was above the mean level for their age and those with substantial cognitive impairment or dementia (Ngandu et al., 2014).

The large population-based study ensures sufficient power and good generalizability of results to older adults at-risk. The amount of data collected also allowed for adjusting for many possible confounding factors. In assessing neuropsychological performance we used a comprehensive and standardized NTB comprising of measurements of several critical cognitive domains and psychometrically validated (Crawford et al., 2003; Ronnlund et al., 2008) method for assessing SMCs.

A limitation of the study is the timing of the subjective memory assessment. Participants completed the PRMQ for the first time 6 months after neuropsychological testing. At that point in time, participants in the intervention group had received some dietary advice and the physical-activity intervention had started. However, no major changes in memory functions or their subjective assessments are likely to have occurred during this time period. It may also be an advantage that the experience of the cognitive testing did not immediately precede the evaluations of subjective memory problems. NTB scores applied in the FINGER study covered the most essential cognitive domains (memory, executive functions, and processing speed) typically affected in normal aging, MCI and early dementia. However, it did not cover all possible cognitive domains.

FINGER targeted the at-risk segment of the general elderly population, not patients in a clinical setting. However, due to the long pre-dementia stage, neuropathological alterations may have started (Dubois et al., 2016; Jack et al., 2013), and some participants may already have had dementia-related brain changes, although no one was diagnosed with dementia at baseline. The mean cognitive performance was less than $0.5 S D$ below the average level for the cognitively normal Finnish population. Our results may, therefore, not be generalizable to older persons performing above the mean level or to those who are severely impaired. The Finnish translation of PRMQ was used in this study for the first time. Although there are no previous Finnish validation studies or norms for the Finnish population, PRMQ is widely used internationally and overall can be seen as culturally neutral. Furthermore, the mean PRMQ scores observed in our study resemble closely those found previously (Crawford et al., 2003).

In conclusion, this study clarifies the relationship between SMCs and objective cognitive performance. The results were in line with previous findings on the association between subjective and objective cognitive functioning (Hohman et al., 2011; Jacinto et al., 2014; Steinberg et al., 2013), and indicate that SMCs should be taken seriously. They may help to identify persons who require further clinical examination.
SMCs often involve both PM and RM problems and, based on our results, their associations to tests commonly used for measuring the cognitive function may differ. Additionally, processing speed seems to play a significant role in SMCs. It is also important to consider affective factors among people who report SMCs and persons with depressive symptoms should be closely monitored.

\section{ACKNOWLEDGMENTS}

We sincerely thank all participants of the FINGER study. The authors acknowledge the Academy of Finland's Responding to Public Health Challenges Research Programme (SALVE) grants, Academy of Finland 259615, 278457 projects, Academy of Finland for Joint Program of Neurodegenerative Disorders - prevention (MIND-AD); the Finnish Medical Foundation, La Carita Foundation, Alzheimer Association grant (HAT-10-173121), Juho Vainio Foundation, Novo Nordisk Foundation, Finnish Social Insurance Institution, Ministry of Education and Culture Research Grant, and EVO/VTR grants of University Hospitals of Kuopio, Oulu and Turku, Seinäjoki Central hospital and Oulu City Hospital for FINGER project, Swedish Research Council; Alzheimer's Research \& Prevention Foundation USA; AXA Research Fund; the Sheika Salama Bint Hamdan Alahyan Foundation, the Swedish Research Council, the Swedish Research Council for Health, Working Life, and Welfare, an Alexander von Humboldt Research Award, and a donation from the af Jochnick Foundation (LB) as well as personal grants from Yrjö Jahnsson Foundation (6567) (LV), Maire Taponen Foundation (LV) and Finnish Brain Foundation (LV). We thank all members of the FINGER study group for their cooperation in data collection and management. The authors declare no conflict of interest. FINGER Study Group: Main investigator: prof. Miia Kivipelto. Coordinator: PhD Tiia Ngandu, MSc Jenni Lehtisalo. Sub-cohort leaders: prof. Miia Kivipelto, prof. Tiina Laatikainen, prof. Hilkka Soininen, prof. Timo Strandberg, prof. Riitta Antikainen, prof. Antti Jula, and prof. Jaakko Tuomilehto. Statistical analyses: MSc Esko Levälahti. Intervention supervision: PhD Jaana Lindström and MSc Jenni Lehtisalo (nutrition component), prof. Rainer Rauramaa and PhD Satu Pajala (physical exercise component), $\mathrm{PhD}$ Tuomo Hänninen and $\mathrm{PhD}$ Tiia Ngandu (cognitive component), prof. Timo Strandberg, prof. Riitta Antikainen and prof. Jaakko Tuomilehto (vascular risk factor component). Other study members and collaborators: Prof. Markku Peltonen, PhD Alina Solomon, MA Psy Teemu Paajanen, MSc Marko Grönholm, Ms Liisa Saarikoski, PhD Francesca Mangialasche.

\section{REFERENCES}

Aalto, A. M., Aro, A. R., \& Teperi, J. (1999). RAND-36 as a measure of health-related quality of life. reliability, construct validity, and reference values in the Finnish general population. Stakes, Research Reports: Helsinki, Finland, 101.

Alzheimer's Disease International. (2015). World Alzheimer report 2015: The global impact of dementia. London: Alzheimer's Disease International.

Amariglio, R. E., Becker, J. A., Carmasin, J., Wadsworth, L. P., Lorius, N., Sullivan, C., ... Rentz, D. M. (2012). Subjective cognitive complaints and amyloid burden in cognitively normal older individuals. Neuropsychologia, 50(12), 2880-2886. 
Aribisala, B. S., Royle, N. A., Maniega, S. M., Valdés Hernández, M. C., Murray, C., Penke, L., ... Wardlaw, J. M. (2014). Quantitative multi-modal MRI of the Hippocampus and cognitive ability in community-dwelling older subjects. Cortex, 53, 34-44.

Bisiacchi, P., Tarantino, V., \& Ciccola, A. (2008). Aging and prospective memory: The role of working memory and monitoring processes. Aging Clinical and Experimental Research, 20(6), 569-577.

Buckley, R., Saling, M., Ellis, K., Rowe, C., Maruff, P., Macaulay, L. S, ... Ames, D. (2015). Self and informant memory concerns align in healthy memory complainers and in early stages of mild cognitive impairment but separate with increasing cognitive impairment. Age Ageing, 44(6), 1012-1019.

Crawford, J. R., Smith, G., Maylor, E. A., Della Sala, S., \& Logie, R. H. (2003). The prospective and retrospective memory questionnaire (PRMQ): Normative data and latent structure in a large non-clinical sample. Memory, 11(3), 261-275.

Crumley, J. J., Stetler, C. A., \& Horhota, M. (2014). Examining the relationship between subjective and objective memory performance in older adults: A meta-analysis. Psychology \& Aging, 29(2), 250-263.

De La Vega, F., Lazaruk, K., Rhodes, M., \& Wenz, M. (2005). Assessment of two flexible and compatible SNP genotyping platforms: TaqMan ${ }^{\circledR}$ SNP genotyping assays and the SNPlex ${ }^{\mathrm{TM}}$ genotyping system. Mutation Research, 573(1), 111-135.

Dubois, B., Hampel, H., Feldman, H. H., Scheltens, P., Aisen, P., Andrieu, S., ... Jack, C. R Jr. (2016). Preclinical Alzheimer's disease: Definition, natural history, and diagnostic criteria. Alzheimer's \& Dementia, 12, 292-323.

Eschen, A., Martin, M., Gasser, U. S., \& Kliegel, M. (2009). Prospective and retrospective memory complaints in mild cognitive impairment and mild Alzheimer's disease. Brain Impairment, 10(1), 59-75.

Finkel, D., McArdle, J. J., Reynolds, C. A., \& Pedersen, N. L. (2007). Age changes in processing speed as a leading indicator of cognitive aging. Psychology \& Aging, 22(3), 558-568.

Folstein, M. F., Folstein, S. E., \& McHugh, P. R. (1975). "Mini-mental state". A practical method for grading the cognitive state of patients for the clinician. Journal of Psychiatric Research, 12(3), 189-198.

Golden, C. (Ed.) 1978. Stroop color and word test: A manual for clinical and experimental uses. Chicago, IL: Skoelting.

Hänninen, T., Pulliainen, V., Sotaniemi, M., Hokkanen, L., Salo, J., Hietanen, M., ... Erkinjuntti, T. (2010). Early detection of cognitive changes in memory diseases: New cut-off scores for the Finnish version of CERAD neuropsychological battery. Duodecim, 126, 2013-2021. (in Finnish).

Hänninen, T., Reinikainen, K. J., Helkala, E. L., Koivisto, K., Mykkänen, L., Laakso, M., ... Riekkinen, P. J. (1994). Subjective memory complaints and personality traits in normal elderly subjects. Journal of the American Geriatrics Society, 42(1), 1-4.

Harrison, J., Minassian, S., Jenkins, L., Black, R., Koller, M., \& Grundman, M. (2007). A neuropsychological test battery for use in Alzheimer disease clinical trials. Archives of Neurology, 64(9), 1323-1329.

Hohman, T. J., Beason-held, L. L., Lamar, M., \& Resnick, S. M. (2011). Subjective cognitive complaints and longitudinal changes in memory and brain function. Neuropsychology, 25(1), $125-130$.

Iliffe, S., \& Pealing, L. (2010). Subjective memory problems. British Medical Journal, 340(7748), 703-706.
Jack, C. R, Knopman, D. S, Jagust, W. J., Petersen, R. C, Weiner, M. W., Aisen, P. S., ... Trojanowski, J. Q. (2013). Tracking pathophysiological processes in Alzheimer's disease: An updated hypothetical model of dynamic biomarkers. The Lancet Neurology, 12(2), 207-216.

Jacinto, A., Brucki, S., Porto, C., Martins, M., \& Nitrini, R. (2014). Subjective memory complaints in the elderly: A sign of cognitive impairment? Clinics, 69(3), 194-197.

Jessen, F., Feyen, L., Freymann, K., Tepest, R., Maier, W., Heun, R., ... Scheef, L. (2006). Volume reduction of the entorhinal cortex in subjective memory impairment. Neurobiology of Aging, 27(12), 1751-1756.

Jones, S., Livner, Å, \& Bäckman, L. (2006). Patterns of prospective and retrospective memory impairment in preclinical Alzheimer's disease. Neuropsychology, 20(2), 144-152.

Jorm, A. F., Christensen, H., Korten, A. E., Jacomb, P. A., \& Henderson, A. S. (2001). Memory complaints as a precursor of memory impairment in older people: A longitudinal analysis over 7-8 years. Psychological Medicine, 31(3), 441-449.

Jorm, A. F., Butterworth, P., Anstey, K. J., Christensen, H., Easteal, S., Maller, J., ... Sachdev, P. (2004). Memory complaints in a community sample aged 60-64 years: Associations with cognitive functioning, psychiatric symptoms, medical conditions, APOE genotype, hippocampus and amygdala volumes, and white-matter hyperintensities. Psychological Medicine, 34(8), 1495-1506.

Jungwirth, S., Fischer, P., Weissgram, S., Kirchmeyr, W., Bauer, P., $\&$ Tragi, K. (2004). Subjective memory complaints and objective memory impairment in the Vienna-Transdanube aging community. Journal of the American Geriatrics Society, 52(2), 263-268.

Kivipelto, M., Ngandu, T., Laatikainen, T., Winblad, B., Soininen, H., Tuomilehto, J. (2006). Risk score for the prediction of dementia risk in 20 years among middle aged people: A longitudinal, population-based study. Lancet Neurol, 5(9), 735-741.

Kivipelto, M., Solomon, A., Ahtiluoto, S., Ngandu, T., Lehtisalo, J., Antikainen, R., ... Soininen, H. (2013). The Finnish geriatric intervention study to prevent cognitive impairment and disability (FINGER): Study design and progress. Alzheimer's and Dementia, 9(6), 657-665.

Kliegel, M., \& Jager, T. (2006). Can the prospective and retrospective memory questionnaire (PRMQ) predict actual prospective memory performance? Current Psychology, 25(3), 182-191.

Kliegel, M., \& Martin, M. (2003). Prospective memory research: Why is it relevant? International Journal of Psychology, 38(4), 193-194.

Kliegel, M., \& Zimprich, D. (2005). Predictors of cognitive complaints in older adults: A mixture regression approach. European Journal of Ageing, 2(1), 13-23.

Kuznetsova, K. A., Maniega, S. M., Ritchie, S. J., Cox, S. R., Storkey, A. J., Starr, J. M., ... Bastin, M. E. (2016). Brain white matter structure and information processing speed in healthy older age. Brain Structure \& Function, 221, 3223-3235.

Larrabee, G., \& Crook, T. (1994). Estimated prevalence of ageassociated memory impairment derived from standardized tests of memory function. International Psychogeriatrics, 6(1), 95-104.

Lee, P. (2014). The relationship between memory complaints, activity and perceived health status. Scandinavian Journal of Psychology, 55(2), 136-141.

Lehrner, J., Moser, D., Klug, S., Gleis, A., Auff, E., Dal-Bianco, P., ... Pusswald, G. (2014). Subjective memory complaints, 
depressive symptoms and cognition in patients attending a memory outpatient clinic. International Psychogeriatrics, 26(3), 463-473.

Lemke, U., \& Zimprich, D. (2005). Longitudinal changes in memory performance and processing speed in old age. Aging, Neuropsychology \& Cognition, 12(1), 57-77.

Mäntylä, T. (2003). Assessing absentmindedness: Prospective memory complaint and impairment in middle-aged adults. Memory \& Cognition, 31(1), 15-25.

Martin, M., Kliegel, M., \& Mcdaniel, M. (2003). The involvement of executive functions in prospective memory performance of adults. International Journal of Psychology, 38(4), 195-206.

Maylor, E. A., Smith, G., Della Sala, S. D., \& Logie, R. H. (2002). Prospective and retrospective memory in normal aging and dementia: An experimental study. Memory \& Cognition, 30(6), 871-884.

McDaniel, M. A., \& Einstein, G. O. (2011). The neuropsychology of prospective memory in normal aging: A componential approach. Neuropsychologia, 49(8), 2147-2155.

Meiberth, D., Scheef, L., Wolfsgruber, S., Boecker, H., Block, W., Traber, F., ... Jessen, F. (2015). Cortical thinning in individuals with subjective memory impairment. Journal of Alzheimer's Disease, 45(1), 139-146.

Mitchell, A. J., Beaumont, H., Ferguson, D., Yadegarfar, M., \& Stubbs, B. (2014). Risk of dementia and mild cognitive impairment in older people with subjective memory complaints: Meta-analysis. Acta Psychiatrica Scandinavica, 130(6), 439-451.

Mol, M., Carpay, M., Ramakers, I., Rozendaal, N., Verhey, F., \& Jolles, J. (2007). The effect of perceived forgetfulness on quality of life in older adults; a qualitative review. International Journal of Geriatric Psychiatry, 22(5), 393-400.

Montejo, P., Montenegro, M., Fernandez, M. A., \& Maestu, F. (2011). Subjective memory complaints in the elderly: Prevalence and influence of temporal orientation, depression and quality of life in a population-based study in the city of Madrid. Aging \& Mental Health, 15(1), 85-96.

Morris, J. C. (1988). Consortium to establish a registry for Alzheimer's disease (CERAD) clinical and neuropsychological assessment of Alzheimer's disease. Psychopharmacology Bulletin, 24(4), 641-652.

Ngandu, T., Lehtisalo, J., Levalahti, E., Laatikainen, T., Lindstrom, J., Peltonen, M., ... Kivipelto, M. (2014). Recruitment and baseline characteristics of participants in the Finnish geriatric intervention study to prevent cognitive impairment and disability (FINGER)-A randomized controlled lifestyle trial. International Journal of Environmental Research and Public Health, 11(9), 9345-9360.

Ngandu, T., Lehtisalo, J., Solomon, A., Levälahti, E., Ahtiluoto, S., Antikainen, R., ... Kivipelto, M. (2015). A 2 year multidomain intervention of diet, exercise, cognitive training, and vascular risk monitoring versus control to prevent cognitive decline in at-risk elderly people (FINGER): A randomised controlled trial. Lancet, 385(9984), 2255-2263.

Owsley, C., Sloane, M., McGwin, G. Jr, \& Ball, K. (2002). Timed instrumental activities of daily living tasks: Relationship to cognitive function and everyday performance assessments in older adults. Gerontology, 48(4), 254.

Penke, L., Muñoz Maniega, S., Murray, C., Gow, A. J., Valdés Hernández, M. C., Clayden, J. D., ... Deary, I. J. (2010). A general factor of brain white matter integrity predicts information processing speed in healthy older people. Journal of Neuroscience, 30(22), 7569-7574.

Piauilino, D. C., Bueno, O. F. A., Tufik, S., Bittencourt, L. R., SantosSilva, R., Hachul, H., ... Pompeia, S. (2010). The prospective and retrospective memory questionnaire: A population-based random sampling study. Memory, 18(4), 413-426.

Ponds, R., \& Jolles, J. (1996). Memory complaints in elderly people: The role of memory abilities, metamemory, depression, and personality. Educational Gerontology, 22(4), 341-357.

Reid, L. M., \& MacLullich, A. M. J. (2006). Subjective memory complaints and cognitive impairment in older people. Dementia \& Geriatric Cognitive Disorders, 22(5), 471-485.

Reitan, R. (1958). Validity of the trail making test as an indicator of organic brain damage. Perceptual and Motor Skills, 8(7), 271-276.

Rönnlund, M., Vestergren, P., Mäntylä, T., \& Nilsson, L. (2011). Predictors of self-reported prospective and retrospective memory in a population-based sample of older adults. Journal of Genetic Psychology, 172(3), 266-284.

Ronnlund, M., Mantyla, T., \& Nilsson, L. (2008). The prospective and retrospective memory questionnaire (PRMQ): Factorial structure, relations to global subjective memory ratings, and Swedish norms. Scandinavian Journal of Psychology, 49(1), 11-18.

Rosnick, C. B., Small, B. J., Graves, A. B., \& Mortimer, J. A. (2004). The association between health and cognitive performance in a population-based study of older adults: The Charlotte County Healthy Aging Study (CCHAS). Aging, Neuropsychology \& Cognition, 11(1), 89-99.

Saaristo, T., Peltonen, M., Keinanen-Kiukaanniemi, S., Vanhala, M., Saltevo, J, Niskanen, L., ... Tuomilehto, J. (2007). National type 2 diabetes prevention programme in Finland: FIN-D2D. International Journal of Circumpolar Health, 66 , 101-112.

Salthouse, T. A. (2000). Aging and measures of processing speed. Biological Psychology, 54(1-3), 35-54.

Salthouse, T. A., Berish, D. E., \& Siedlecki, K. L. (2004). Construct validity and age sensitivity of prospective memory. Memory \& Cognition, 32(7), 1133-1148.

Samieri, C., Proust-Lima, C., M. Glymour, M., Okereke, O. I., Amariglio, R. E., Sperling, R. A., ... Grodstein, F. (2014). Subjective cognitive concerns, episodic memory, and the APOE e4 allele. Alzheimer's \& Dementia, 10(6), 752-759.

Sargent-Cox, K., Cherbuin, N., Sachdev, P., \& Anstey, K. J. (2011). Subjective health and memory predictors of mild cognitive disorders and cognitive decline in ageing: The personality and total health (PATH) through life study. Dementia and Geriatric Cognitive Disorders, 31(1), 45-52.

Schneider, J. A., Arvanitakis, Z., Leurgans, S. E., \& Bennett, D. A. (2009). The neuropathology of probable Alzheimer's disease and mild cognitive impairment. Annals of Neurology, 66(2), 200-208.

Small, G. W., Chen, S. T., Komo, S., Ercoli, L., Bookheimer, S., Miller, K., ... Pericak-Vance, M. A. (1999). Memory selfappraisal in middle-aged and older adults with the apolipoprotein E-4 allele. American Journal of Psychiatry, 156(7), $1035-1038$.

Steinberg, S. I., Negash, S., Sammel, M. D., Bogner, H., Harel, B. T., Livney, M. G., ... Arnold, S. E. (2013). Subjective memory complaints, cognitive performance, and psychological factors in healthy older adults. American Journal of Alzheimer's Disease and Other Dementias, 28(8), 776-783. 
Stewart, R., Russ, C., Richards, M., Brayne, C., Lovestone, S., \& Mann, A. (2001). Depression, APOE genotype and subjective memory impairment: A cross-sectional study in an AfricanCaribbean population. Psychological Medicine, 31(3), 431-440.

Studer, J., Donati, A., Popp, J., \& von Gunten, A. (2014). Subjective cognitive decline in patients with mild cognitive impairment and healthy older adults: Association with personality traits. Geriatrics \& Gerontology International, 14(3), 589-595.

Van der Elst, W., Van Boxtel, M. P., Van Breukelen, G. J., \& Jolles, J. (2006a). The concept shifting test: Adult normative data. Psychological Assessment, 18, 424-432.

Van der Elst, W., van Boxtel, M. P., van Breukelen, G. J., \& Jolles, J. (2006b). The letter digit substitution test: Normative data for 1,858 healthy participants aged 24-81 from the Maastricht Aging Study (MAAS): Influence of age, education, and sex. Journal of Clinical and Experimental Neuropsychology, 28(6), 998-1009.

Vartiainen, E., Laatikainen, T., Peltonen, M., Juolevi, A., Mannisto, S., Sundvall, J., ... Puska, P. (2010). Thirty-five-years in cardiovascular risk factors in Finland. International Journal of Epidemiology, 39, 504-518.

Woods, S. P., Weinborn, M., Velnoweth, A., Rooney, A., \& Bucks, R. S. (2012). Memory for intentions is uniquely associated with instrumental activities of daily living in healthy older adults. Journal of the International Neuropsychological Society, 18(1), 134-138.

Wechsler, D. (Ed.) (1998). WMS-III-Administration and scoring manual (Psychological Corporation Ltd ed.). London, UK: Psychological Corporation Ltd.

Wilson, B. A., Cockburn, J., \& Baddeley, A. D. (Eds.) (1985). The Rivermead Behavioural Memory Test. London: Pearson Assessment.

Zeintl, M., Kliegel, M., Rast, P., \& Zimprich, D. (2006). Prospective memory complaints can be predicted by prospective memory performance in older adults. Dementia \& Geriatric Cognitive Disorders, 22(3), 209-215.

Zung, A. (1965). A self-rating depression scale. Archives of General Psychiatry, 12, 63-70. 